Revue des patrimoines

\title{
L'aile dite « des prélats » au château de Grignan (Drôme) 1684-1689
}

\section{Christian Trézin}

\section{(2) OpenEdition}

Journals

Édition électronique

URL : http://journals.openedition.org/insitu/1203

DOI : 10.4000/insitu.1203

ISSN : 1630-7305

Éditeur

Ministère de la culture

Référence électronique

Christian Trézin, «L'aile dite « des prélats » au château de Grignan (Drôme) 1684-1689 », In Situ [En ligne], 2 | 2002, mis en ligne le 23 avril 2012, consulté le 03 mai 2019. URL : http:// journals.openedition.org/insitu/1203; DOI : 10.4000/insitu.1203

Ce document a été généré automatiquement le 3 mai 2019.

\section{(c) $($ i) $(9)$}

In Situ Revues des patrimoines est mis à disposition selon les termes de la licence Creative Commons Attribution - Pas d'Utilisation Commerciale - Pas de Modification 4.0 International. 


\section{L'aile dite " des prélats » au château de Grignan (Drôme) 1684-1689}

Christian Trézin

\section{Le contexte historique}

Le château de Grignan

Figure 1

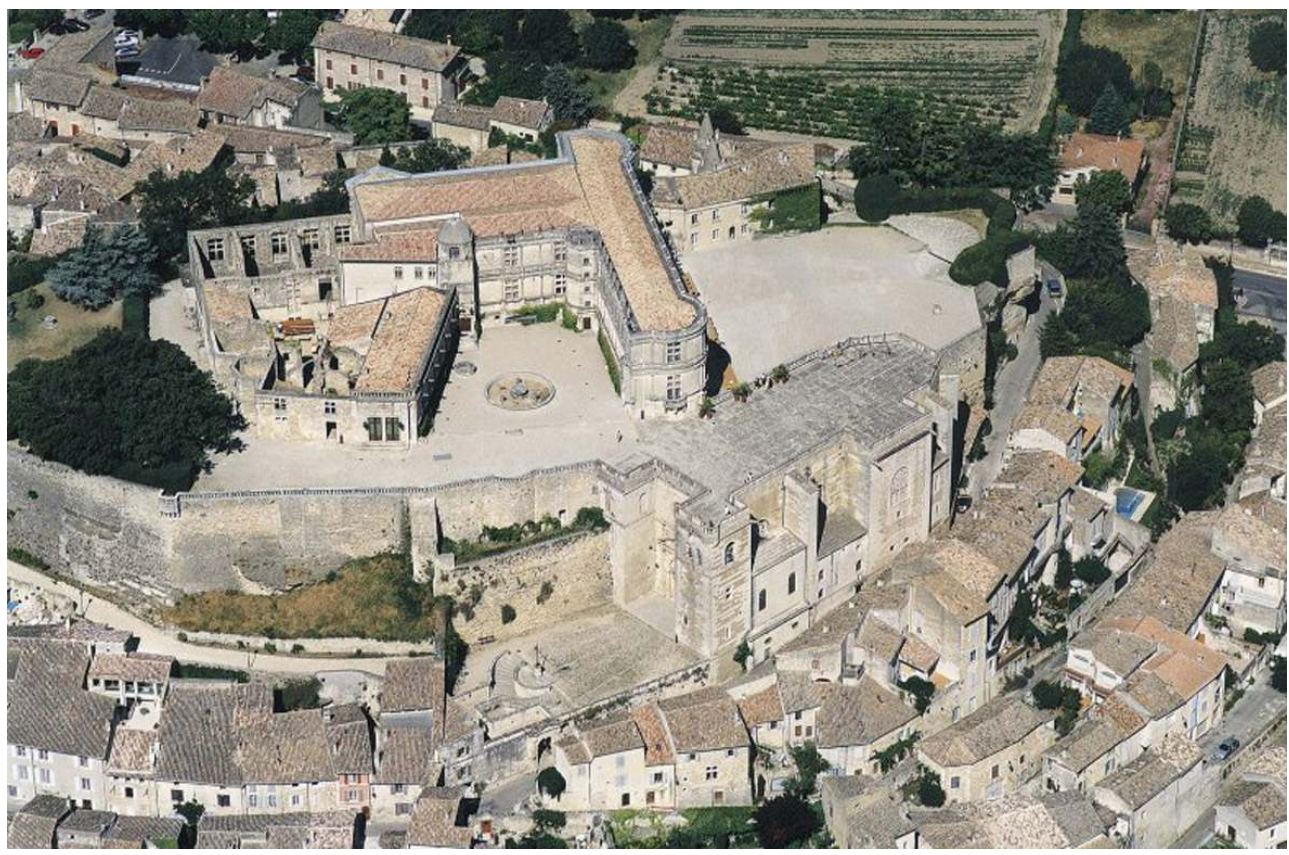

Vue aérienne prise de l'ouest

Phot. Inv. A. Franchella ( Inventaire général, ADAGP, 2001 
1 Après l'apparition vers l'an 1000 du castellum qu'un texte atteste en 1035, le promontoire qui porte le château de Grignan a été peu à peu aménagé : modelage des escarpements, enceinte, fortification d'une plate-forme dominant la basse-cour et probablement entourée de défenses. Cette topographie s'imposa à l'implantation des bâtiments et à la structure du château fort qui s'y développa du XIII ${ }^{\mathrm{e}}$ au XV $\mathrm{XV}^{\mathrm{e}}$ siècle $^{1}$. Ce que l'on peut en connaître pour la fin du Moyen Age montre à quel point les bâtisseurs des siècles suivants eurent peu de marge d'initiative face à cette organisation initiale, contraints à la fois par la présence des constructions médiévales et par l'exiguïé du site.

2 Le château connaît plusieurs phases principales de développement: au XIII ${ }^{\mathrm{e}}$ siècle construction d'un châtelet d'entrée et d'un corps de salle dans le prolongement d'une tour carrée antérieure (XII siècle ?) ; renfort des accès au XIV ${ }^{\mathrm{e}}$ siècle (tours de la poterne occidentale et renfort du châtelet par la grosse tour semi-circulaire accotée à la tour carrée). Le corps de salle et les deux tours qui au sud s'y rattachent alors forment aux XIV ${ }^{e}$ et $\mathrm{XV}^{\mathrm{e}}$ siècles l'essentiel du logis seigneurial.

3 La cour du puits est le cadre des premières phases de modernisation au dernier quart du $\mathrm{XV}^{\mathrm{e}}$ siècle. Après 1478 Gaucher Adhémar, baron de Grignan, double en profondeur le corps de salle pour compléter le logis seigneurial. Il construit vers 1495-1515 une galerie reliée à ce dernier par un large escalier droit voûté d'ogives, adossé au corps de logis. Entre 1540 et 1558 son fils Louis Adhémar, , fait orner ce dernier et l'agrandit au sud d'un corps en retour. Il fait aussi remanier la chapelle Saint-Romain et les communs au nord de celle-ci, et bâtir contre le flanc ouest du site l'église collégiale Saint-Sauveur (1535-1542) couverte d'une terrasse dallée rendue accessible depuis la cour du puits².

4 A la suite des chantiers du XVI ${ }^{\mathrm{e}}$ siècle le front est du château devait ainsi présenter le résultat de la juxtaposition progressive, du sud au nord, de la tour carrée (XII e siècle ?), de la tour renforçant le châtelet (XIV siècle), du corps de salle (XIII ${ }^{\mathrm{e}}$ siècle), du corps contenant l'escalier droit (1495-1515), enfin de constructions mal définies que les vestiges d'un passage voûté (Moyen Âge), d'un couvrement d'ogives (1495-1515 ?) et la présence de murs de refend et d'une cheminée en anse de panier (2e moitié du XVI ${ }^{\mathrm{e}}$ siècle-1er quart du XVII siècle ?) laissent présumer. Dans les années 1684-1689 François de Castellane-Adhémar fait bâtir à cet emplacement l'aile « des prélats » dont nous traitons ici, venant clore l'évolution architecturale de l'édifice.

5 Par son arrêté du 1er nivôse an II (20 décembre 1793) l'administration du district de Montélimar ordonne la démolition du château de Grignan. Précédée du 17 au 27 décembre de la vente du mobilier, son exécution en janvier 1794 se limite au démantèlement de l'édifice et à la destruction partielle de la façade sud sur la basse-cour ${ }^{3}$. Seules les intempéries dégraderont l'aile "des prélats». Le pillage qui s'ensuit est interrompu par le rachat en 1838 par Léopold Faure (AD Drôme, notaire Laurens, Montélimar), puis poursuivi par le comte Boni de Castellane après 1902 jusqu'à son acquisition en 1912 par Marie Fontaine, née Barroux de Heerdt (AD Drôme, notaire Misson, Grignan). 


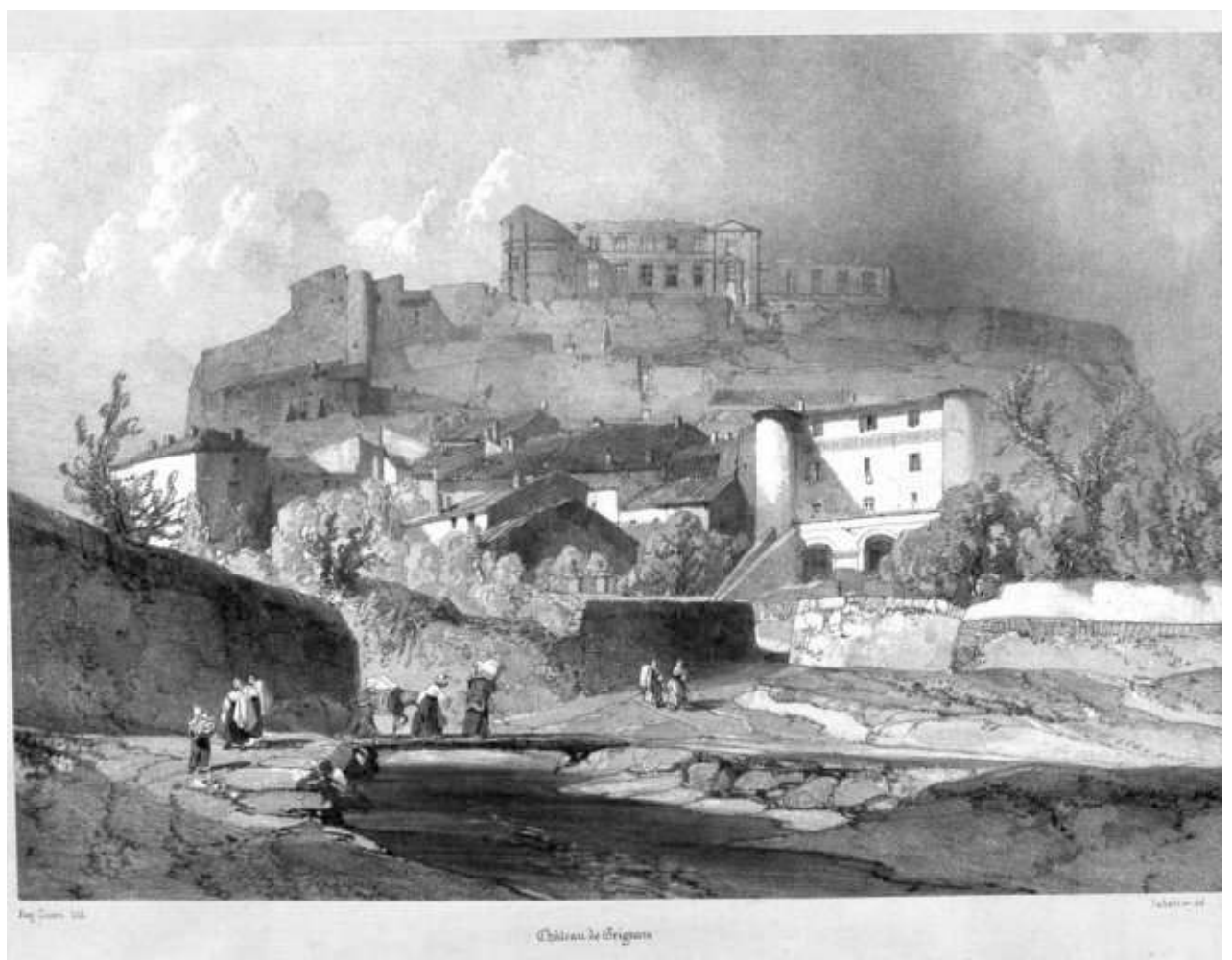

"Château de Grignan »

Taylor, Baron J., Nodier, C., Cailleux, A. de, Voyages pittoresques et romantiques dans I'ancienne France. Paris : Gide fils, 1820-1881, 23 vol. (Dauphiné, t. 2, 1835). Sabatier del, Ciceri Eugène lith [Lemercier imp Paris]. (c) Conseil général de la Drôme, 1997

\section{Les commanditaires après 1668}

6 François de Castellane-Adhémar, héritier du comté de Grignan en 1668, épouse FrançoiseMarguerite de Sévigné. Louis XIV le nomme en 1669 lieutenant-général au gouvernement de Provence avec les fonctions de gouverneur. Il meurt en 1714 après avoir été pendant quarante-cinq ans le premier personnage de Provence.

7 Ses difficultés financières ${ }^{4}$ le conduisent à demander l'aide de ses frères pour le chantier de l'aile est qu'il entreprend à partir de 1684. Louis-Joseph est évêque de Carcassonne en 1681 après avoir occupé le siège d'Evreux. Jean-Baptiste a été depuis 1667 coadjuteur de son oncle François, archevêque d'Arles, puis titulaire de cette dignité en $1689^{5}$. Il avait amené les consuls d'Arles à confier en 1673 à Jules Hardouin-Mansart la construction de l'hôtel de ville, chantier qu'il suivit de près 6 .

\section{L'aile « des prélats »}

\section{Les circonstances de son aménagement}

Dès 1671 Mme de Sévigné témoigne du projet : il s'agit « d'achever de bâtir » le château, allusion probable à l'inachèvement de chantiers antérieurs (Duchene, I, 183, 19 juillet $1671)^{7}$. 
9 Le château modernisé et agrandi au XVI ${ }^{e}$ siècle s'était avéré pendant un siècle conforme aux besoins d'un grand seigneur. Mais le pouvoir étendu de François de CastellaneAdhémar impliquait une vie de cour à laquelle ne répondaient plus vraiment ni l'allure « antique » du château, ni le nombre, ni la distribution de ses appartements, lorsque le comte devait recevoir à Grignan "tous ces trains qui passaient, ces compagnies sans compte et sans nombre qui se faisaient un air d'y aller de toutes les provinces " (Duchene, II, 769, 31 mai 1680 ; III, 1093, 4 avril 1689). Ainsi se trouve justifiée la profonde transformation de la partie est.

Mme de Sévigné témoigne encore que cette construction ne vit pas le jour sans peine. Les travaux privaient le comte d'une bibliothèque, « lui ôtant les logements des étrangers et des domestiques » et laissant une "halle de salon (...) tout ouvert et tout dégradé » (Duchene, III, 1128, et 1147, 17 juillet et 14 septembre 1689). «L'air mortel de Maintenon » (Duchene, III, 1011, 18 octobre 1688) régnait ici lorsque les ouvriers modelaient le rocher ou taillaient les pierres de construction, emplissant de poussière un air rendu irrespirable par la grâce des vents.

\section{La construction de 1684 à 1690}

11 Le fonds d'Alauzier conservé aux archives départementales du Vaucluse comprend un dossier de pièces relatives à ce chantier dont le doyen du chapitre de la collégiale SaintSauveur de Grignan, Joseph de Ripert d'Alauzier, était le mandataire pour le compte de Louis-Joseph de Castellane-Adhémar (43 J 142)

12 Le «prix-fait... pour l'apartement de Mgr de Carcassonne au château de Grignan [est] donné à maître Florent Loizeleur, masson », le 6 octobre 1684 « suivant le dessin qu'il en a fait ... » (doc. L). La pierre de taille y est prévue pour les seules façades est et nord, la façade ouest devant être en « moilons » crépis et réglés, toutes restant unies à l'exception du larmier de l'appui des fenêtres de l'étage.

D'octobre à août 1685 (doc. A), les travaux ont pour objet de «demoulir le cartier ou monseigneur de carcassonne veu faire batir » (doc. N) ainsi que « la voutte et la muraille du coste de bize de la lingerie... » (doc. L). On retire « les tuiles et le bois qui estoient dans la lingerie et (...) les pierres enterrées dans la ruine... » (doc. A).

Un nouveau prix-fait du 20 avril 1685 (doc. L) prévoit « l'augmentation de l'ouvrage (...) scavoir d'un cordon qui doit régner (...) au dessous de l'appui des fenêtres, d'un balcon au bout du cabinet (...), plus des deux cheminées de la sale et de la garderobbe ». La comptabilité établie en 1687 montre qu'un surplus de travaux a concerné le «taleu dudepuis le fondement jusque sous le cordon» des façades est et nord et le « revestissement de la taille de la muraille du coste du couchant depuis le boudin jusques a l'appuy » (doc. L).

15 La pose de la première pierre a lieu en août 1685 (doc. B). Le bâtiment est probablement élargi vers la cour du four à l'ouest, d'où nécessité de "pétarder" les rochers en décembre 1685 et janvier 1686 " pour faire les fondements » de la muraille du salon. La fouille (1983-1984) a en effet montré le creusement du rocher au pied de la façade occidentale. Les voûtes des "offices basses » sont étayées et terrassées en novembre et décembre 1686 (doc. B).

Les quatre façades sont construites depuis le fondement (doc. L). La façade nord reste dépourvue de pignon alors que sont mentionnés ceux des deux refends et du mur sud. 
17 Le gros œuvre semble achevé avant le printemps 1687. Florent Loyseleur disparaît après septembre 1686. La fourniture de tuiles en septembre 1686 et de poutres début février 1687 (doc. B) laissent à penser que la couverture est en place dans les mois qui suivent, d'autant que les portes et fenêtres, dont le prix fait est baillé au menuisier Mézangeau, de Bollène, en octobre 1686 (doc. B et $\mathrm{P}$ ), sont achevées et payées le 4 mai 1687 (doc. P).

Un autre maçon, Jean-Baptiste Bousquet, originaire de Pont-Saint-Esprit ${ }^{9}$, est chargé de travaux de second œuvre comme les voûtes en tuf du salon et le "pavé » selon un prix fait qui lui est donné le 17 juillet 1687 (doc. M). Leur construction est en cours en septembre (doc. B et M). Le 24 janvier 1688, un prix fait est donné au menuisier Mézangeau pour les "corniches » à faire "conformement au paneau que Mr Mignard donnera signé et parraffé de sa main » (doc. P). Un compte établit après avril 1688 que le premier " plafons du salon de la chambre passage et cabinet » a été fait puis que l'on a "abbatu le plafons du salon» et fait «le second plafons du salon» (doc. M). Notre documentation sur le second œuvre du bâtiment de Monsieur de Carcassonne s'interrompt alors pendant près d'un an.

Dans l'intervalle, vers octobre 1688 ou peu avant, démarre la construction de l'archevêque d'Arles dont les conséquences se feront sentir ultérieurement sur le chantier de Carcassonne. Le témoignage de Mme de Sévigné, dans l'esprit d'une chronique, constitue notre seule source. «Vous me représentez le bâtiment de Monsieur de Carcassonne comme un vrai corps sans âme, manquant d'esprit et surtout du nerf de la guerre. Je pense que le Coadjuteur n'en manque pas moins. Eh, mon Dieu ! que veulent-ils faire?» (Duchene, III, 1015, 26 octobre 1688) ; «Je vois que le bâtiment du Coadjuteur ira bien ... mais celui de Carcassonne vous tourmentera tout l'été » (Duchene, III, 1017, 1er novembre 1688) ; "J'ai foi à l'envie qu'a le Coadjuteur d'achever son bâtiment, mais j'en ai encore pluis à la longueur infinie de celui de Monsieur de Carcassonne » (Duchene, III, 1021, 8 novembre 1688); «Je conjure ... M. le Coadjuteur d'achever son bâtiment ... la honte qu'il y aurait à ne pas achever ce qui est commencé » (Duchene, III, 1022, 10 novembre 1688) ; «Vos prélats vous ont quitté. J'admire toujours également celui qui fait bâtir, et celui qui n'achève point son bâtiment... » (Duchene, III, 1045, 22 décembre 1688) ; «... l'état affreux de votre château... par la fureur de M. le Coadjuteur, aussi préjudiciable que le tourbillon. Quelle rage est la sienne de bâtir et de débâtir... » (Duchene, III, 1070, 14 février 1689); "Mais je vois bien que mon complot ne vaudra rien, et qu'il entend Mansart qui l'appelle, et qu'il ne dira point adieu à sa chère truelle. Et Carcassonne laissera-t-il la sienne en l'air ? » (Duchene, III, 1105, 2 mai 1689).

Les travaux de Monsieur de Carcassonne ont repris vers avril 1689. Avant le 4 mai il est à nouveau question de fenêtres que le menuisier Mézangeau est en train de faire (doc. D et P) et il est "payé aux perreyeurs a compte des nouvelles croisées » (doc. D). C'est au maçon Jacque Jacquet dit Beaufleury qu'est baillé avant le 15 mai 1689 un « prixfait (...) des fenestres et fassade » et le « second [prixfait] du balcon ». Les manœuvres sont payés en août du travail «pour les fenêtres ou les cintres » (doc. D). Les comptes de 1689 font également apparaître ce qui «est deu a mezangeau ... pour huit fenestres... pour le rabillage des portes et fenêtres » (doc. $P$ ).

En mai-juin s'effectuent le crépissage de diverses pièces (petite et grande chambre, passage), la préparation de la pose de « [la corniche] du salon et de la chambre sur les modeles de celle du salon et de la chambre de Mr de la Garde » (doc. D), le vitrage des fenêtres (doc. D et $\mathrm{O}$ ), la mise en place du « plancher de la pettite chambre et du passage » (entresol) (doc. U). D'autres menus travaux sont payés en juillet (doc. D et Q). 

trouverions du bois pour faire la charpente de notre dernier étage, et qu'ainsi M. d'Arles verrait son appartement habitable...mais comment peut faire M. de Carcassonne de résister à la vivacité de $\mathrm{M}$. d'Arles, qui prend le lièvre au corps en lui disant : 'Donnez-moi 400 écus et rendormez-vous et laissez-moi faire' ?» (Duchene, III, 1167. L'appartement d'Arles est partiellement compris dans le bâtiment de Carcassonne). Mais dès le 16 novembre Mme de Sévigné « loue (...) M. l'Archevêque d'avoir le courage d'achever son bâtiment, et (...) l'admire d'avoir obtenu 400 écus de M. de Carcassonne» (Duchene, III, 1167). Cette somme apparaitra dans la comptabilité finale d'avril 1690 comme ayant été laissée à Grignan par le doyen de Ripert à Avril, intendant du comte de Grignan. Le compte de « François Bouschet [pour] l'entier payement du couvert de l'hautvent de Mgr de Carcassonne » intervient après le 26 décembre. L'apurement des comptes est achevé en avril 1690.

\section{Après le XVIII siècle}

Léopold Faure acquéreur du château en 1838 interrompt par quelques toitures provisoires le processus de dégradation initié en 1794. Le comte Boni de Castellane nourrit dit-on un projet de restauration ${ }^{11}$ mais dépouille notamment la grande salle de sa cheminée.

Marie Fontaine, pour l'essentiel entre 1913 et 1920, réalise la restauration qui reste partielle pour l'aile "des prélats » : elle s'attache à sa moitié sud, à son corps central, et au rez-de-chaussée de sa partie nord dont l'étage reste en ruine. Les principales 
distributions sont conservées mais les aménagements et décors intérieurs, disparus ou trop dégradés, sont reconstitués. Conseillée par un proche, Mgr Meffre, elle confie la conduite des travaux à l'architecte Julien de L'Isle-sur-la-Sorgue (Vaucluse) et les décors au ferronnier Zalaffi de Sienne, au peintre Ratto et au sculpteur Endignoux d'Avignon (inscriptions).

Lors de sa cession au conseil général de la Drôme en 1979, plusieurs des décors sont déposés par les héritiers de Marie Fontaine. Un réaménagement les a remplacés, adaptant l'existant dans une perspective muséographique achevée en 1987.

\section{Les caractéristiques du bâtiment}

\section{Le programme}

Traditionnellement qualifié d'«aile des prélats », le programme de ce chantier, justifié par une recherche de cohésion architecturale, n'est pas la construction d'une aile nouvelle. Plus simplement il érige devant l'ensemble des corps de bâtiments des siècles passés une façade écran qui les masque et les complète.

Figure 3

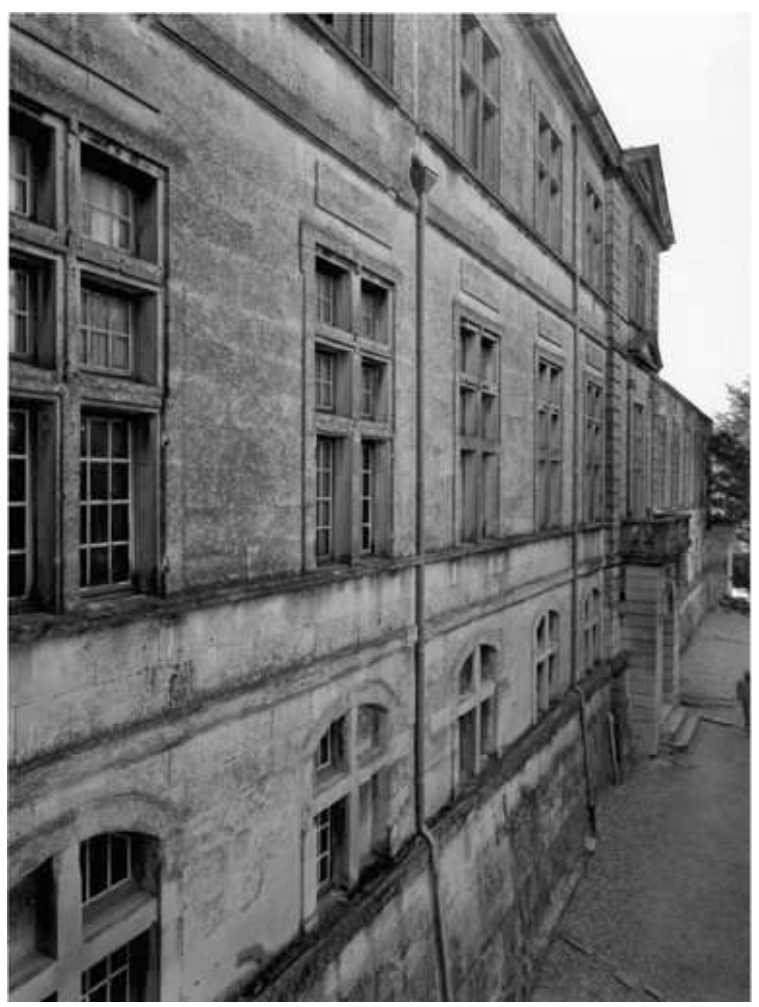

Vue générale de l'aile « des prélats », prise vers le nord

Phot. Inv. A. Franchella @ Inventaire général, ADAGP, 1998 


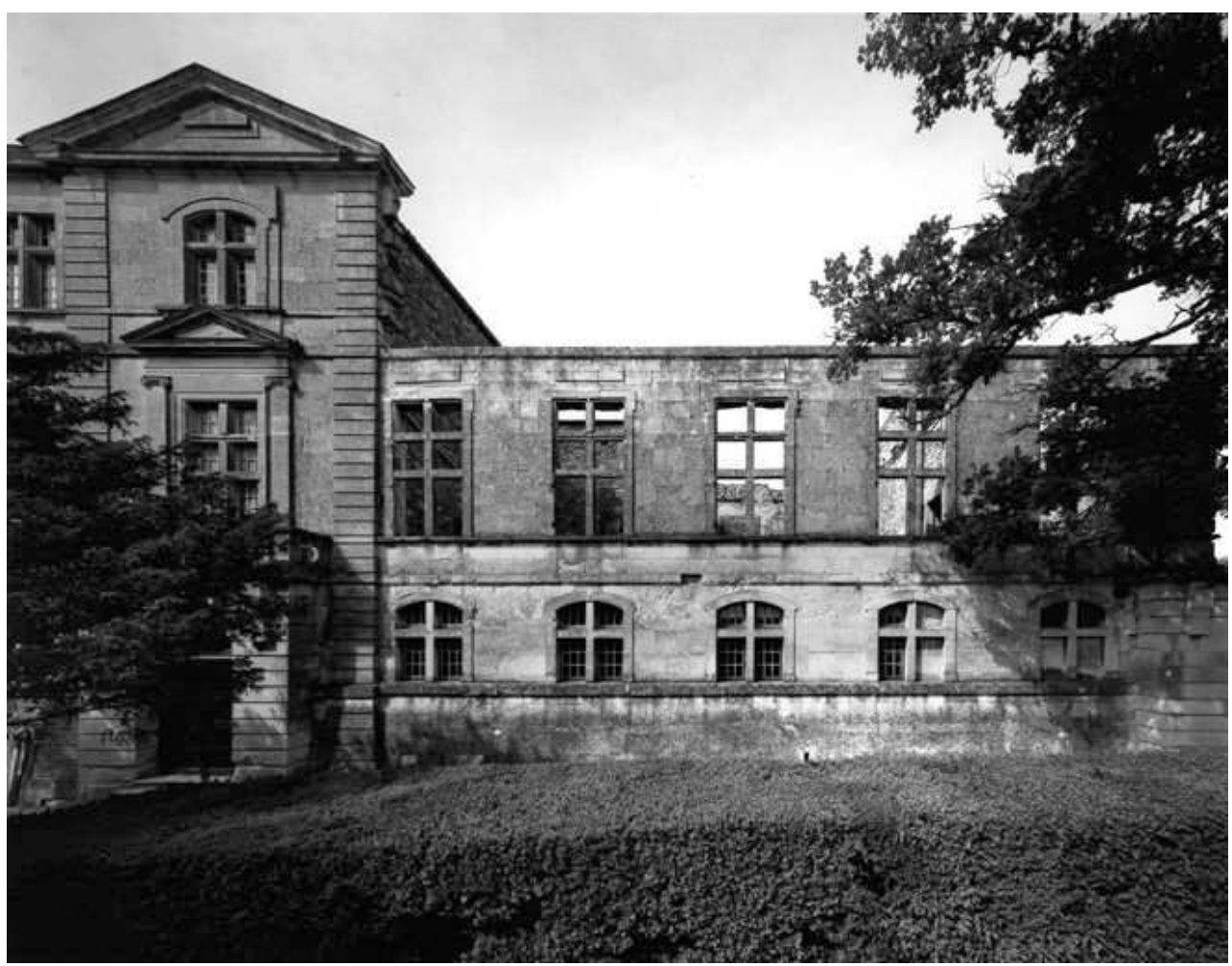

Vue de l'aile « des prélats ». Le bâtiment de Carcassonne

Phot. Inv. A. Franchella ( Inventaire général, ADAGP, 1998

Du même coup il modernise l'aspect du château, permet un réaménagement de l'existant et la création de superficies nouvelles. On y distingue vers le sud le bâtiment d'Arles intégrant le corps central, et celui de Carcassonne vers le nord.

\section{Les matériaux}

La pierre de taille est fournie par les «perreyeurs» de Chamaret (Fuoc, Borromée, La Violette, Splandian) comme l'indiquent les sources (P. C, D, E et N). Il s'agit d'un calcaire molassique miocène proche dans sa composition et son apparence de la pierre de SaintRestitut. Elle est mise en œuvre en moyen appareil réglé du côté sud, et en moyen appareil réglé alterné de module allongé du côté nord. Un bossage continu en table est employé pour le talus de la façade orientale et pour les chaînages d'angle marquant les extrémités de l'aile et les angles du corps central.

\section{La structure}

Derrière l'apparente unité architecturale recherchée par le projet, les relevés reflètent la genèse progressive du secteur. Cet ensemble complexe de pièces hétérogènes et mal articulées est un héritage qui ne saurait être attribué aux travaux des années 1684-1689. Du côté sud notamment le bâtiment est double en profondeur, résultat du développement postérieur à 1478, alors qu'il est simple au nord. Par ailleurs le projet architectural de 
l'archevêque d'Arles n'ayant pas été relayé dans toute son ampleur par $M$. de Carcassonne, le nombre de niveaux est resté limité à deux du côté nord.

Figure 5

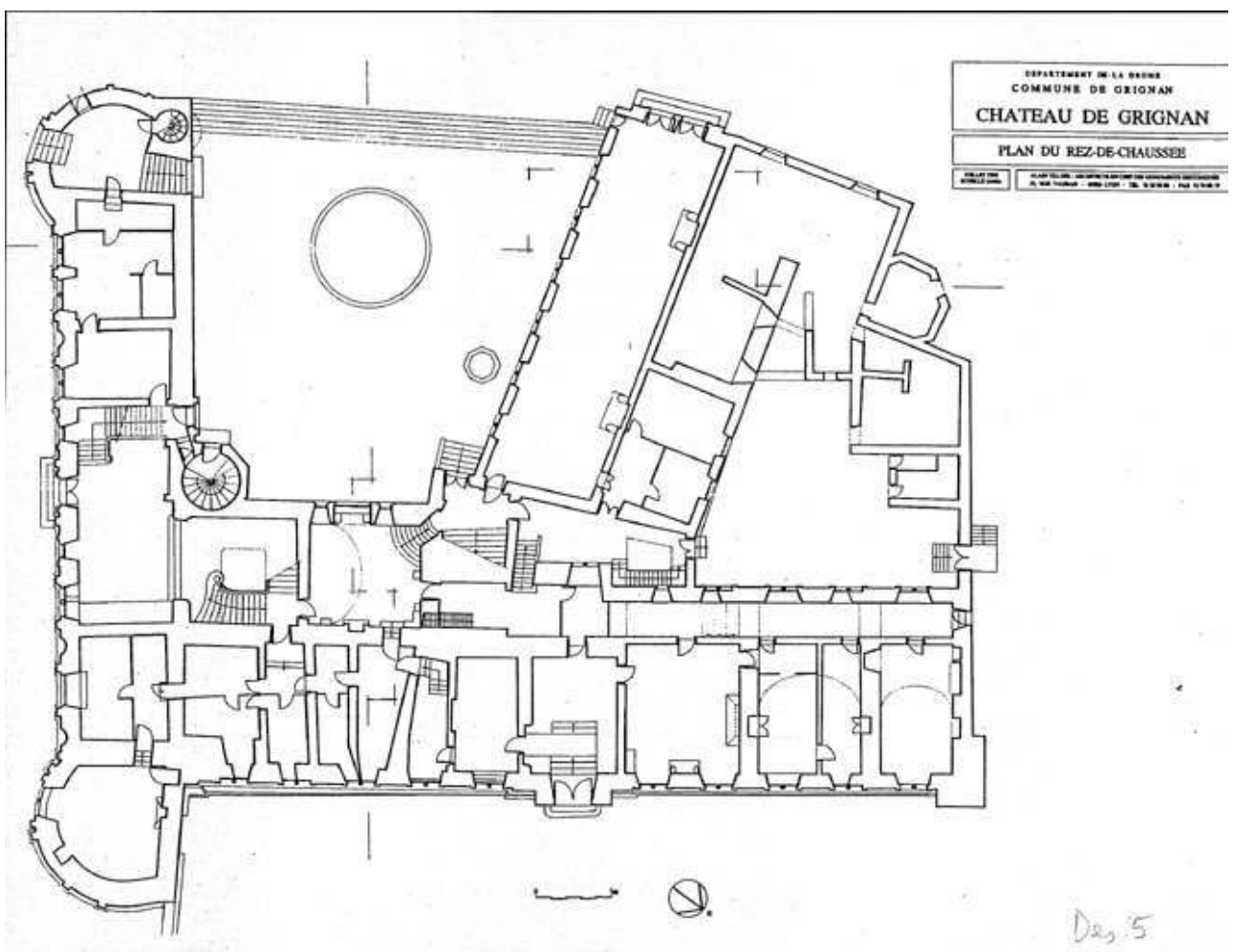

Plan du rez-de-chaussée du château de Grignan

(c) Alain Tillier, 1995 


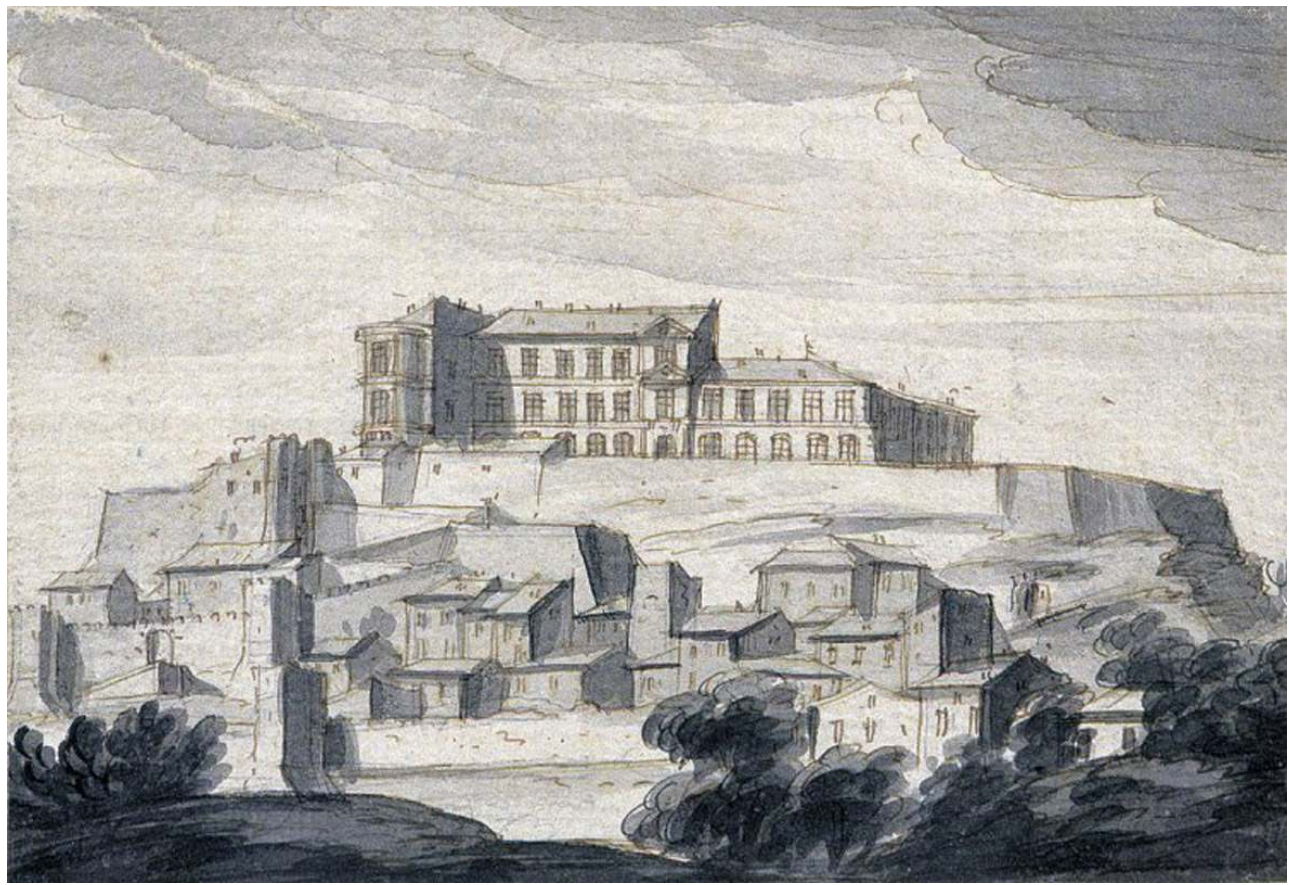

"Vue de Grignan au levant. D'après nature »

Dessin à l'encre et lavis (après 1694), collection Musée du château de Grignan. (ancienne collection Puech, Avignon) @ Conseil général de la Drôme, 1997

Les niveaux inférieurs sont voûtés dans leur ensemble et l'irrégularité des murs témoigne des reprises et des remaniements.

Figure 7

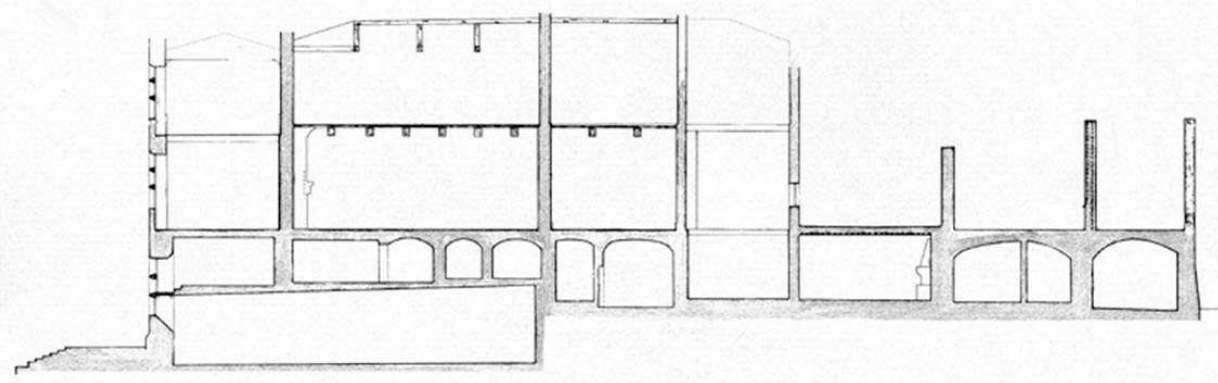

Coupe longitudinale sud-nord

(c) Christian Trézin, 1999

Le bâtiment d'Arles reflète clairement les dispositions médiévales. L'archéologie a montré que la surépaisseur considérable de ses niveaux inférieurs est due au placage en 1689 du parement de pierre de taille en talus sur la base du corps de salle médiéval. A la voûte longitudinale de la grande cave se superposent de petites pièces irrégulières en position d'entresol. Les refends parfois obliques y obturent deux baies. L'une des voûtes est longitudinale, les autres transversales. 
Le niveau inférieur du bâtiment de Carcassonne forme en revanche un ensemble plus régulier. Le talus ne provoque qu'une simple surépaisseur et la façade nord atteint $1, ?$ ?m . Les refends y montrent cependant quelques épaisseurs supérieures aux façades est et ouest et l'un d'eux vient ici encore obturer une baie, témoignant de dispositions antérieures au chantier des années 1684-1689. Le couloir est voûté sur doubleaux, deux pièces ont des voûtes segmentaires, la troisième l'a vraisemblablement perdue après 1793 et ses voûtains datent de la restauration des années 1913-1920.

Figure 8

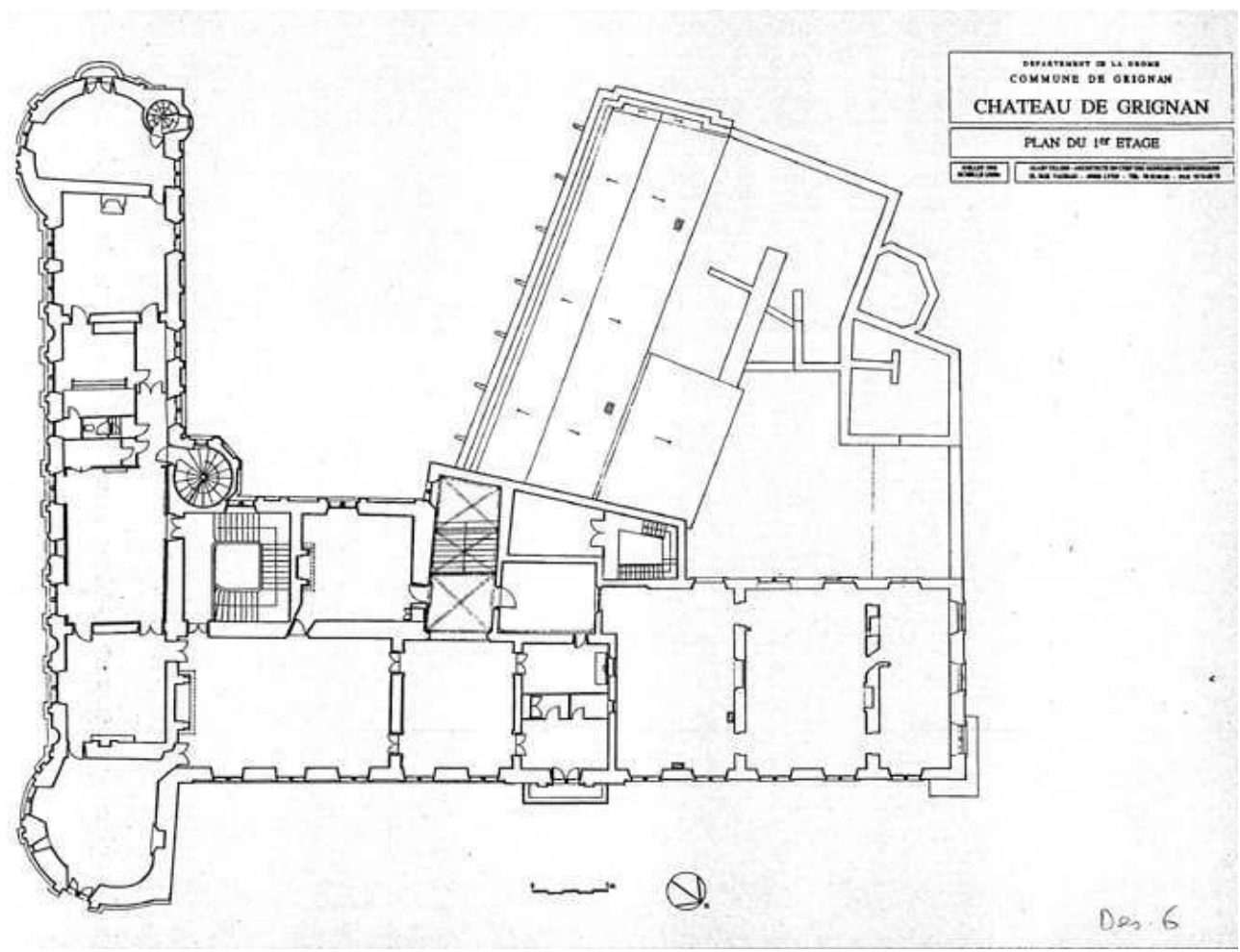

Plan du 1er étage du château de Grignan

(c) Alain Tillier, 1995

L'étage-noble paraît plus homogène d'un bout à l'autre de l'aile. A partir de ce niveau le mur médiéval du bâtiment d'Arles a été rebâti de la même épaisseur que les façades est et ouest du bâtiment de Carcassonne. La structure générale de la partie sud est reprise au dernier étage.

Le corps central est charpenté à chaque étage.

\section{Les élévations}

Les causes de la complexité du plan expliquent aussi celles des élévations. Si la façade orientale de l'ensemble présente un semblant d'unité, il n'en est pas de même à l'ouest où le bâtiment ne possède une volumétrie propre que sur la cour du four. Pour le reste il se raccorde à l'existant.

La façade orientale est la plus représentative de l'ambition architecturale des commanditaires. 


\begin{tabular}{|lcr|}
\hline $26 \quad$ Grignan & \\
Château, aile des prélats & $1: 200$ & \\
Elévation de la façade est. & 00260014NUD \\
Dess. Inv. M. Andry & @inventaire général - Centre $\quad 2000$ \\
\hline
\end{tabular}
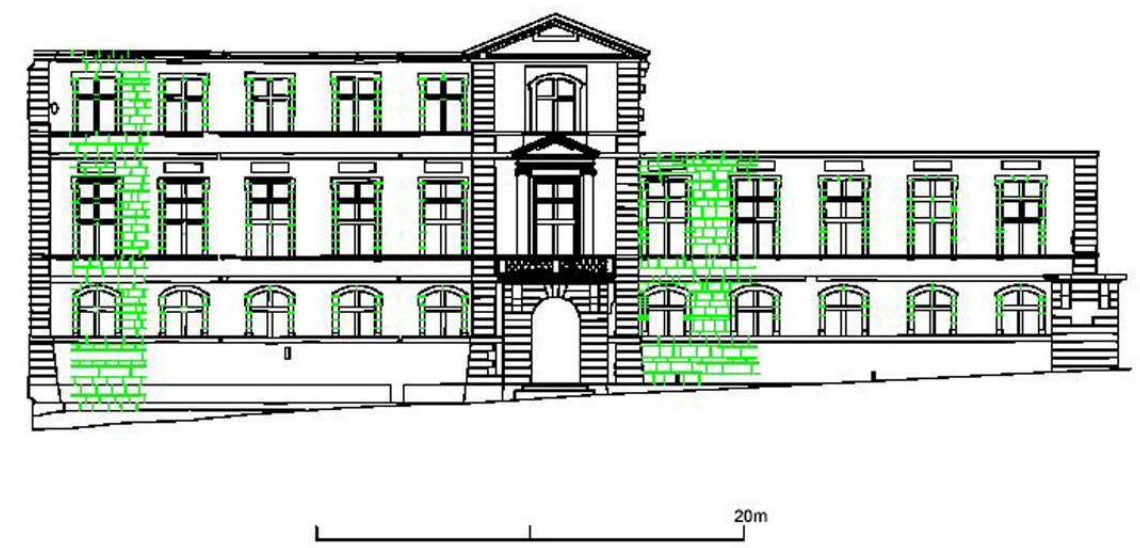

26 Grignan. Château, aile des prélats. Elévation de la façade est

Dess. Inv. M. Andry ( ) Inventaire général - Centre, 2000

41 nord, sur un soubassement taluté amorti par un bandeau. La déclivité du terrain accentue la hauteur du soubassement qui atteint au sud une hauteur considérable englobant le niveau de la cave. A l'angle nord-est, le balcon est porté par un puissant massif inachevé à bossage continu.

Les deux ailes de cinq travées de fenêtres sont organisées autour d'un corps central. Elles combinent selon une formule courante un étage inférieur de petites baies d'entresol en arc segmentaire et un ou deux niveaux de baies à doubles croisées. Toutes les baies ont un chambranle à crossettes hautes et plinthe. Celles de l'étage noble, de proportion plus élancée, sont amorties en table saillante. Les niveaux sont séparés par un double corps de moulures. Les gouttes sous l'appui au droit du chambranle sont groupées par cinq. Une corniche à gargouilles en forme de masque couronne l'aile nord.

Le corps central, partie la plus ornée de l'ensemble, possède un portail en plein cintre ouvert dans un massif à bossage continu dont les consoles en attente d'un décor supportent un balcon autrefois à «balustrade de fer " ${ }^{12}$, aujourd'hui à balustres de pierre carrés. A l'étage noble la baie ouvrant sur le balcon dans un ordre de pilastres ioniques est surmontée d'un fronton triangulaire. L'ouvrage est couronné, au-dessus de la baie en arc segmentaire du troisième niveau, d'un grand fronton triangulaire dont la table attend aussi l'ornement prévu. 
Figure 10

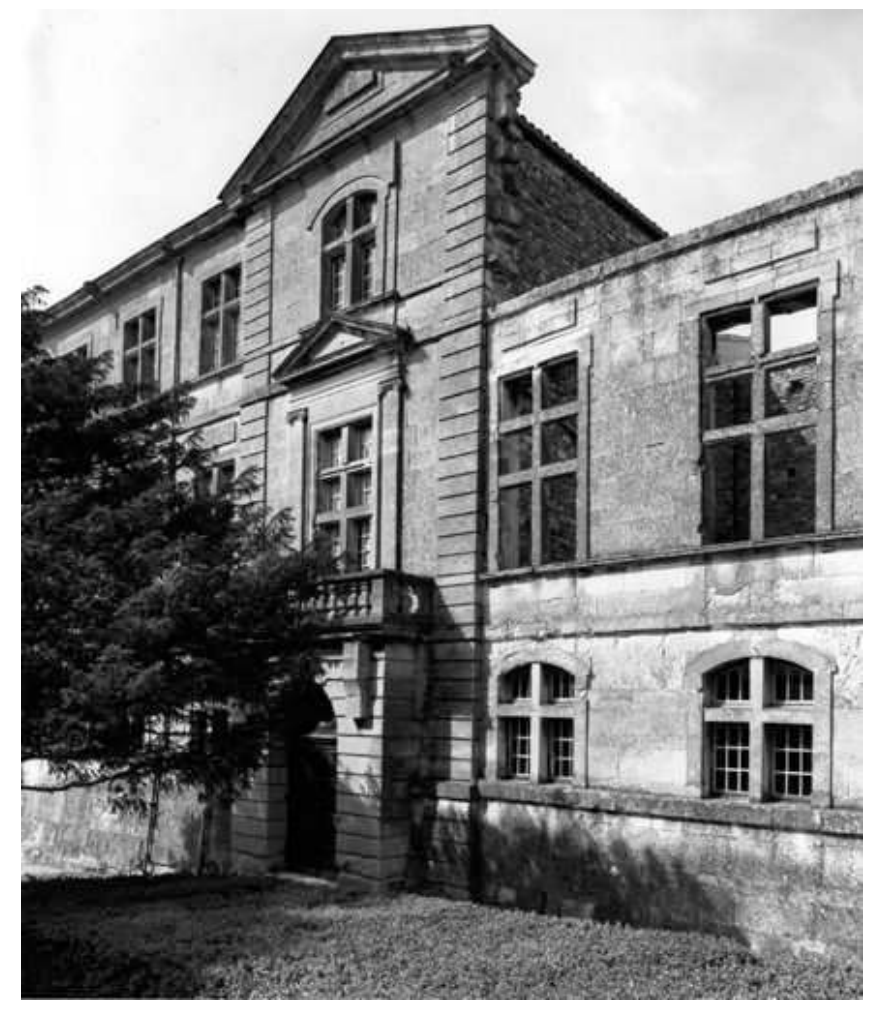

Vue du corps central de l'aile « des prélats »

Phot. Inv. A. Franchella (c) Inventaire général, ADAGP, 1998

Figure 11

\begin{tabular}{|lrr|}
\hline $26 \quad$ Grignan & & \\
Château, aile des prélats & & \\
Elévation de la façade ouest. & $1: 100$ & $00260016 \mathrm{NUD}$ \\
Dess. Inv. M. Andry & 9) Inventaire général - Centre & 2000 \\
\hline
\end{tabular}
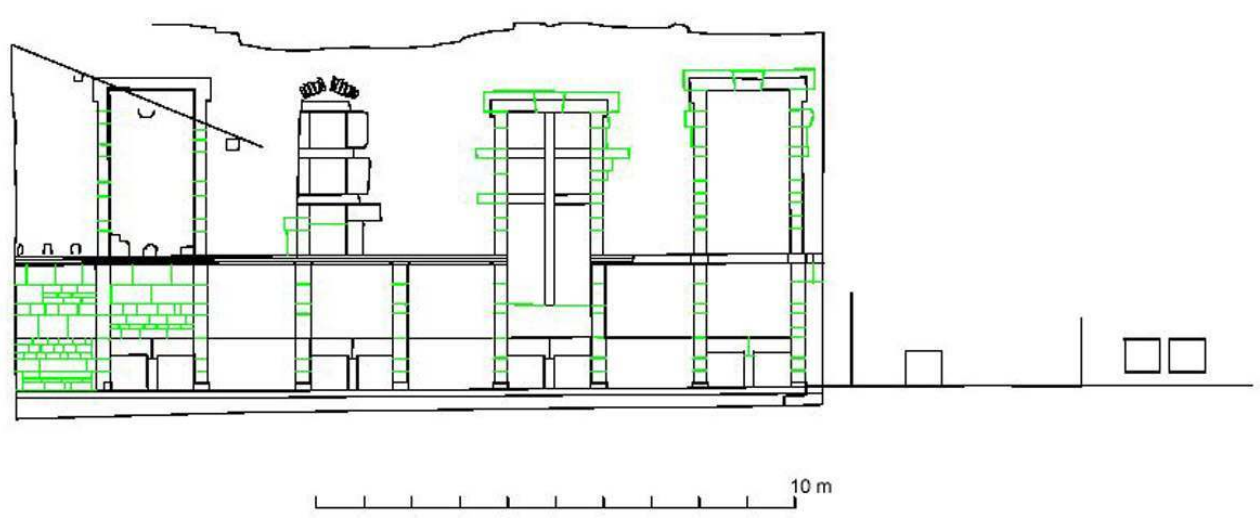

Grignan. Château, aile des prélats. Elévation de la façade ouest

Dess. Inv. M. Andry ( ) Inventaire général - Centre, 2000 
L'analyse des façades ouest et nord est plus complexe. La première n'est visible que de la cour du four. Son soubassement, en moellons jusqu'à hauteur du tore, disparaît sous les remblais qui ont rapproché le sol de la cour du niveau des sols intérieurs du bâtiment qui la limite au sud, et rendu sa clôture nécessaire au nord. Six soupiraux oblongs à platebande et meneau donnent jour au couloir. Seule la partie de façade entre le tore et l'appui des fenêtres est en petit appareil allongé, rythmée par des dosserets qui déterminent huit travées régulières. L'étage est en moellons enduits à faux joints, percé de fenêtres dont quatre seulement restent visibles, trois à double croisée dont une transformée en porte fenêtre, et une demi-croisée, Une seule de ces baies n'a pas été murée par la suite.

Figure 12

\begin{tabular}{|c|c|c|}
\hline \multicolumn{3}{|l|}{26 Grignan } \\
\hline \multicolumn{3}{|c|}{ Château, aile des prélats } \\
\hline Elévation de la façade nord. & $1: 100$ & 00260015 NUD \\
\hline Dess. Inv. M. Andry & (Q) Inventaire géné & Centre 2000 \\
\hline
\end{tabular}
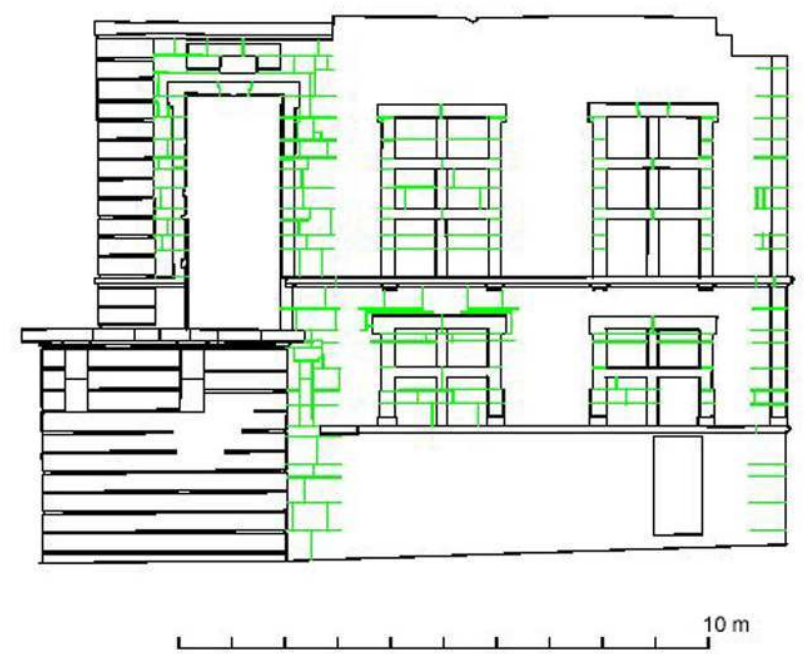

26 Grignan. Château, aile des prélats. Elévation de la façade nord

Dess. Inv. M. Andry (c) Inventaire général - Centre, 2000 


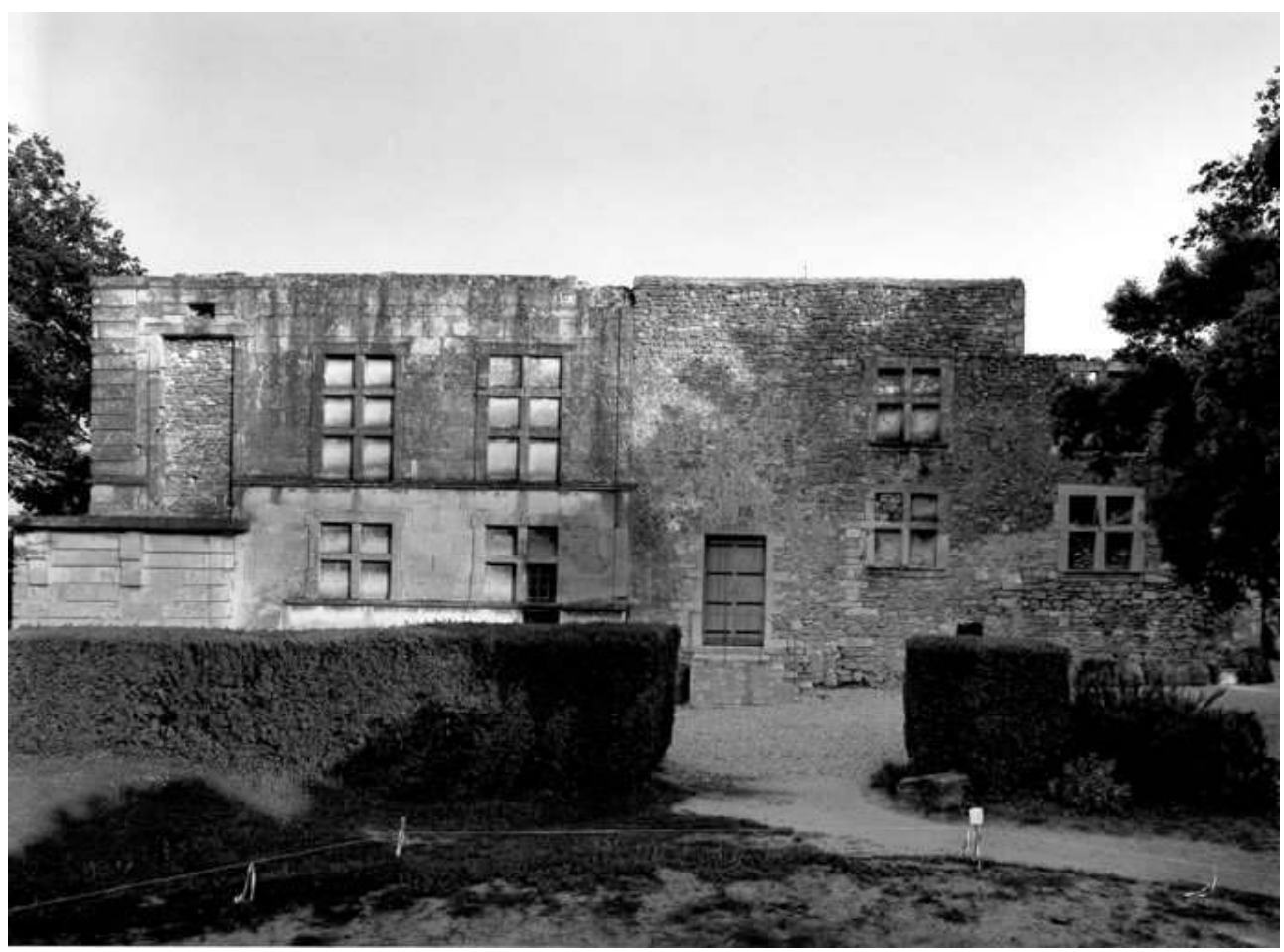

Vue de la façade nord du bâtiment e" Carcassonne

Phot. Inv. A. Franchella ( ) Inventaire général, ADAGP , 1998

La façade nord est en moyen appareil réglé alterné de module allongé sur trois niveaux. Le soubassement taluté est amorti par un tore. Il est percé d'une porte à angles vifs ouvrant sur le couloir et surmonté de deux niveaux.

Le premier, en position d'entresol, est un registre de deux fausses croisées carrées, l'appui au niveau du tore, avec chambranle à peine saillant, crossettes haute et basse, et plinthe. Les crossettes hautes sont formées par l'insertion d'un dé de pierre dans l'angle formé par le débord du linteau. Une seule des baies des deux croisées est ouverte et donne jour au couloir. L'étage noble est percé de trois grandes fenêtres à double croisillon comparables pour le reste à celles de l'entresol, si ce n'est l'absence de crossettes inférieures. Orientées au nord elles ont été murées ultérieurement devant la violence du mistral. Deux sont soulignées à hauteur d'appui par un corps de moulure, orné de gouttes par six au droit des piédroits. L'angle nord-ouest est marqué au-dessus du tore par un dosseret cornier.

A l'angle nord-est le raccord entre les deux façades, par l'intermédiaire du balcon, génère quelques désordres dans les maçonneries et les corps de moulure : le bandeau couronnant la façade nord s'interrompt brutalement près de l'angle et le tore est progressivement bûché jusqu'à disparaître près du balcon. L'ouverture de l'allège de la fenêtre devenue porte-fenêtre du balcon coupe la moulure d'appui. La porte-fenêtre est également rehaussée : on lit la trace des crossettes primitives et les désordres induits dans l'appareil au-dessus du linteau, amorti désormais en table saillante. Au-delà de la porte-fenêtre la moulure d'appui adopte progressivement le profil qu'elle présente à l'est. On remarque enfin à l'est l'absence des dosserets qui rythmaient la façade ouest. 
48 Le relevé photogrammétrique des trois façades montre que le tore de la façade nord, marquant l'amorce du talus, se situe à un niveau supérieur à celui du bandeau de la façade est. Sur cette dernière le niveau correspondant est une assise de même hauteur que le tore, coupant le premier niveau de baies, dont l'observation attentive révèle des traces d'outils distinctes de celles du parement. Cette particularité d'appareillage, absente sur le bâtiment d'Arles, existe sur toute la longueur du bâtiment de Carcassonne où un vestige du talus initial, visible en coupe sous le massif d'angle, apparaît à la même hauteur que sur la façade nord. Le bandeau plat apparaît comme inséré a posteriori dans le parement.

Un examen plus approfondi de la façade est fait apparaître d'autres remaniements du bâtiment de Carcassonne. Au niveau inférieur, pour permettre le logement du cintre des fenêtres, dont les crossettes hautes ne s'alignent pas avec les assises du parement, les plates-bandes, visibles de l'intérieur, ont été réduites en profondeur. Ces mêmes fenêtres ont été agrandies vers le bas. Elles étaient initialement identiques aux soupiraux ouverts dans la façade ouest et ouvraient aussi au niveau du tore.

50 L'appui mouluré de l'étage, bien qu'au même niveau que sur les autres façades, ne présente cependant pas le même profil. Son remaniement progressif visible sur la façade nord aboutit, par simple reprise de taille, à lui conférer son profil de la façade orientale. Comme au niveau inférieur le bandeau plat a été inséré a posteriori dans le parement.

51 Les fenêtres de l'étage ont également été modifiées. Plusieurs présentent encore le dé de pierre qui forme, sous les linteaux de la façade nord, la crossette supérieure de fenêtres de moindre hauteur. Cette surélévation est également lisible dans la présence des tronçons des traverses initiales conservées dans les piédroits.

\section{Les couvertures}

52 La toiture de la partie sud, invisible aujourd'hui, est couverte de tuiles rondes. Elle est à longs pans à croupes avec de faibles pentes. De ce fait la présence d'un simple muret d'attique de faible hauteur suffit à la masquer. A sa base, les gargouilles en forme de masques, évacuent les eaux pluviales recueillies par les noues.

Un document figurant cette aile à la fin du XVII ${ }^{e}$ siècle montre des toitures plus hautes, couvertes de tuiles d'après les sources. Bâties à longs pans à croupe pour le bâtiment de Carcassonne, elles sont en revanche en appentis pour le corps central et le bâtiment d'Arles, appuyées contre le refend longitudinal du logis médiéval dont les documents montrent la surélévation visible du sud comme de l'ouest.

54 L'actuelle charpente à pannes, mise en place peu avant 1920, est sans particularité notable. Il ne reste ni vestige ni document concernant celle du XVII siècle.

\section{Fonctions et communications}

L'ensemble des niveaux inférieurs étaient déjà en $1668^{13}$ destinés aux communs, cuisines et réserves de denrées alimentaires, remplaçant notamment la cuisine qui en 1597 encore se situait dans le corps de bâtiment sud ${ }^{14}$. La complexité fonctionnelle induite par la juxtaposition progressive non concertée de volumes architecturaux se ressent particulièrement à l'examen des communications après les chantiers des années 
1684-1689. Qui plus est la restauration rend parfois difficile la discrimination des divers états de la construction.

L'entrée avec vestibule dans le corps central faisait communiquer la cour des remises avec le niveau des cuisines et des communs. Une volée de marches compense le dénivelé généré par la proximité de la plate-forme que bordent les constructions. Deux ensembles distincts et complémentaires sont identifiés. Au sud les petites pièces consacrées aux réserves (charbonnière, chambre fromagière, charnier, pierres à huile) conservent leur ancienne disposition en enfilade. Au nord le couloir distribue l'ensemble des cuisines, qui communiquent aussi directement entre elles et avec le vestibule. Il se raccorde à un autre, plus ancien, qui, par l'intermédiaire de l'office, assure la liaison avec les réserves et le grand escalier.

On accède à l'étage-noble par ce dernier, mais également par l'escalier droit relié au couloir par quelques marches. Parallèlement à la façade d'Arles se développe après 1689 le principal ensemble du château dont les volumes principaux sont conservés. Les appartements situés au sud et à l'ouest ouvrent sur la "salle neuve ", héritière de l'aula médiévale, réaménagée en $1687-1689^{15}$. Par le vestibule de l'escalier droit elle communique avec la salle commune aux appartements d'Arles et de Carcassonne, largement amputés aujourd'hui. Par l'escalier droit l'ensemble est aussi relié directement avec la galerie, et au sud avec la « salle vieille » par le palier du grand escalier.

Figure 14

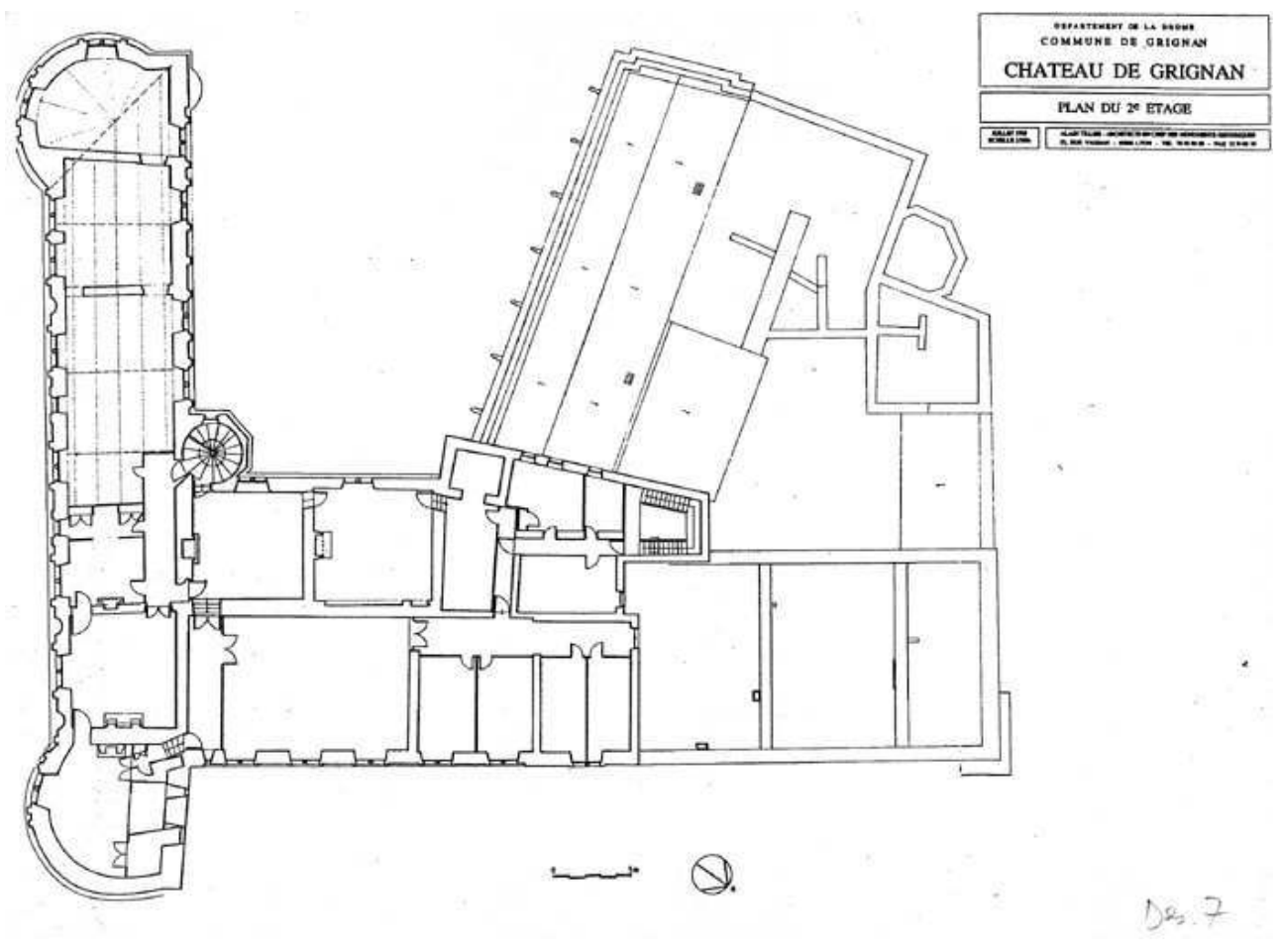

Plan du 2e étage du château de Grignan

(C) Alain Tillier, 1995

L'accès au dernier étage du bâtiment d'Arles affecté lors de sa restauration à l'usage de grenier et de chambres de domestiques, n'est possible aujourd'hui que par un escalier de service en bois à retours au nord, ou par l'escalier en vis du XVI siècle. Celui-ci, partant de la cour du puits, est accessible aussi depuis la salle neuve par le palier du grand 
escalier. A son débouché au dernier étage un vestibule donne sur des couloirs qui distribuent à la fois l'appartement du comte et d'autres espaces répartis au-dessus de la "salle neuve", mais également les autres appartements de la partie ouest du logis médiéval et de l'aile sud. Il est à la fin du XVII ${ }^{e}$ siècle occupé par l'appartement du comte.

\section{Conclusions}

\section{Les défaillances de la maîtrise d'ouvrage} nouvelle dont la vue serait somme toute fort noble, réserve faite de l'aile inachevée. Sa juxtaposition aux constructions voisines est d'une incroyable maladresse. Que ce soit avec le bâtiment de Carcassonne ou avec la tour sud-est et l'aile sur la basse-cour, elle-même modifiée pour recevoir une toiture en appentis vers le sud, les liaisons ne sont pas traitées. Des pignons triangulaires aveugles s'élèvent au-dessus des constructions voisines sans autre forme de procès. Au revers, du côté ouest sur la cour du puits, le refend surélevé du logis, devenu gouttereau et aveugle, domine le versant de toiture de l'autre partie du corps de logis. pas de commentaire, pas même Mme de Sévigné, trop heureuse que s'arrêtent les frais et les désagréments pour sa fille. Seul l'Abbé d'Expilly, qui vit en Grignan «l'une des plus belles antiques de France ", laisse pourtant percer sa réserve, évoquant d'une moue cet « air de nouveauté [donné] au moyen de quelques bâtiments modernes » ${ }^{16}$.

\section{L'œuvre dans son contexte}

\section{Le bâtiment de Carcassonne}

63 Le prix fait du 6 octobre 1684 baillé à Florent Loiseleur lui attribue le dessin du projet. De ce maître maçon on ne connaît que la sacristie de l'église de La Garde-Adhémar où il est mentionné en 1687 comme " tailheur de pierre et entrepreneur de bastimans $~{ }^{17}$. Pendant la durée du chantier de Grignan il est en affaire à la fois avec le marquis de la Garde et 
Monseigneur de Carcassonne (doc. L) qui le paye en février ou mars 1685 pour un travail à la Garde dont nous ignorons la teneur (doc. B).

Les comptes du chantier se réfèrent à des modèles que l'on n'identifie pas toujours clairement. Il n'y a pas d'ambiguité lorsque l'on prévoit le 20 avril 1685 « un balcon au bout du cabinet comme celuy de lappartement de la Reyne ", bâti à Grignan vers 1676 (Duchene, II, 514, 28 mai 1676). En revanche plusieurs références sont obscures : le prix fait du 6 octobre 1684 prévoit des portes et fenêtres « de la même hauteur et largeur que celles de monsieur le Marquis de la Garde »; la note du doyen de Ripert vers mai-juin 1689 (doc. U) commande la corniche « du salon et de la chambre sur les modèles de celle du salon et de la chambre de Mr de la Garde »; le prix fait du 4 octobre 1686 préconise de faire «les quatre grandes portes de l'appartement... avec les mêmes ornements [que] celles de monsieur de Lauzier » (doc. B et P). Quoi qu'il en soit il s'agit de travaux réalisés dans un cercle social limité, à Grignan dont ils sont des familiers, à La Garde-Adhémar ou ailleurs.

La seule référence identifiable en dehors de ce cercle se trouve dans le prix fait donné le 24 janvier 1688 au menuisier Mézangeau pour les corniches du salon, de la chambre et du cabinet qui adopteront un modèle donné par " Mr Mignard ». Il s'agit probablement de Pierre Mignard (1640-1725), neveu du peintre Nicolas Mignard, peintre lui-même et membre de l'Académie royale d'architecture revenu en 1680 s'installer en Avignon.

\section{Figure 15}

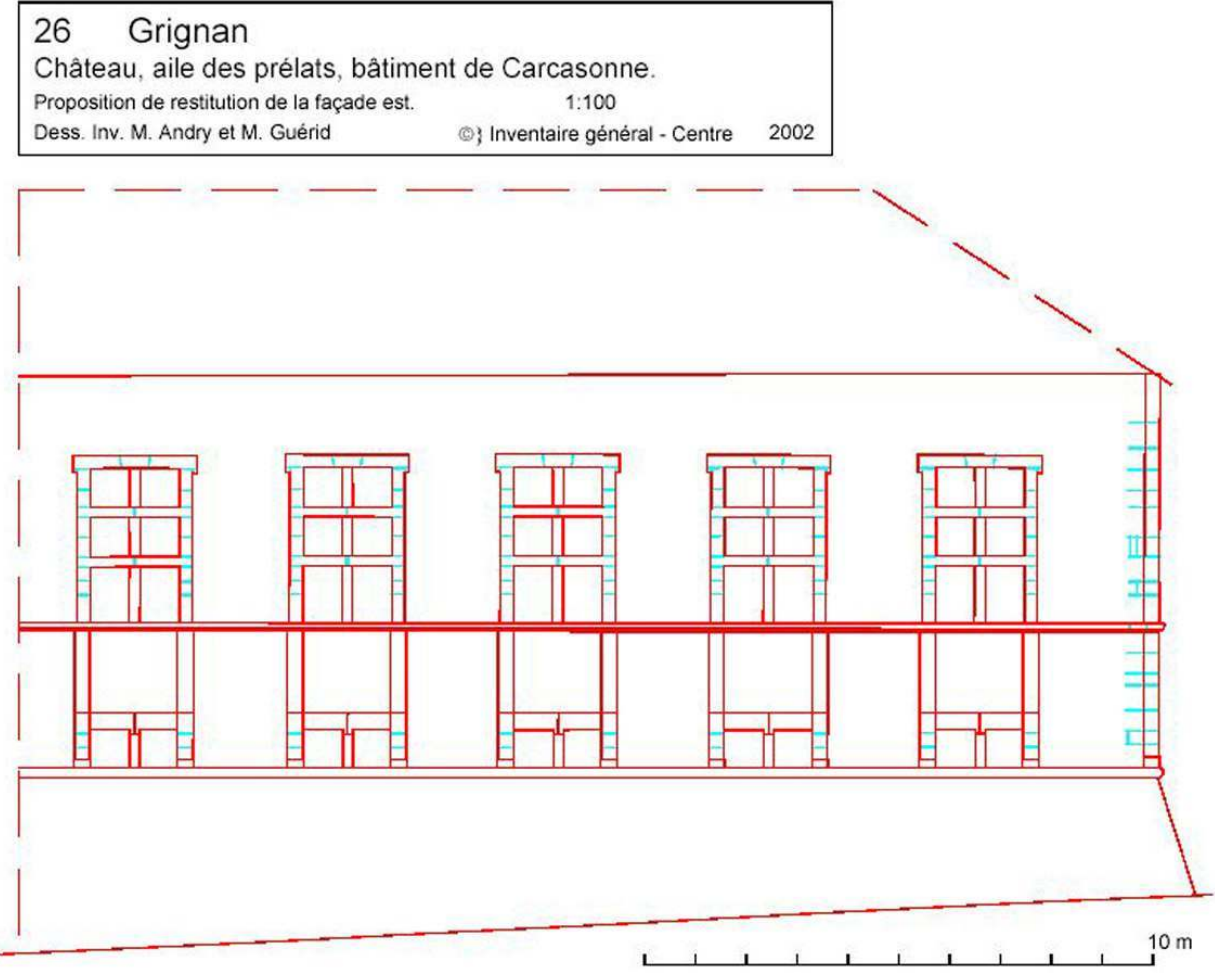

26 Grignan. Château, aile des prélats, bâtiment de Carcasonne. Proposition de restitution de la façade est

Dess. Inv. M. Andry et M. Guérid. (c) Inventaire général - Centre, 2002 

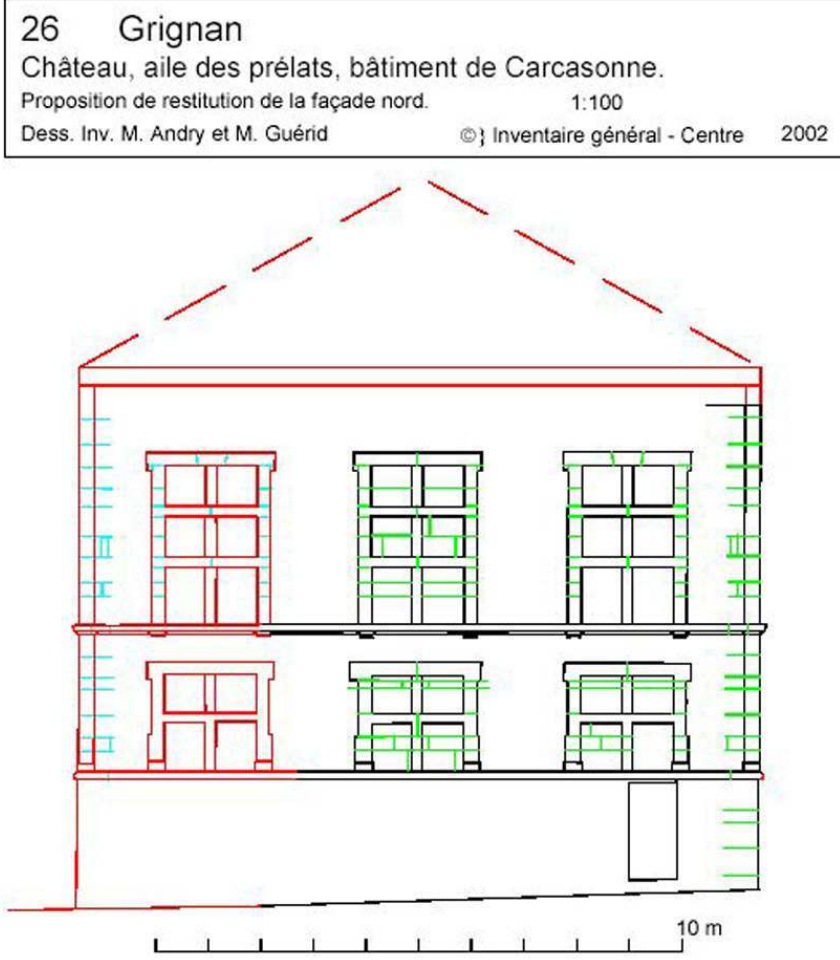

26 Grignan. Château, aile des prélats, bâtiment de Carcasonne. Proposition de restitution de la façade nord

Dess. Inv. M. Andry et M. Guérid. (C) Inventaire général - Centre, 2002

Les observations effectuées sur le bâtiment permettent de restituer un projet initial sans innovation, témoignant dans sa forme générale comme dans son vocabulaire d'un bon savoir-faire d'entrepreneur, issu de plus d'un siècle d'évolution de l'architecture française.

Le soubassement taluté, les deux niveaux de baies animés du simple décor architectural de chambranles à crossettes et d'un discret corps de moulure orné de gouttes, les travées rythmées de dosserets, la volumétrie des toitures hautes à la française évoquent globalement quelques aspects des châteaux sur socle de la seconde moitié du XVI ${ }^{\mathrm{e}}$ siècle aussi bien que les leçons générales du classicisme ou que les hôtels aixois des années 1630-1640 (hôtels de Lacépède, de Roquessante ou Barthélémy).

\section{Le bâtiment d'Arles}

Le déroulement des deux chantiers de Carcassonne et d'Arles avec leurs contradictions et leurs repentirs aboutit cependant pour la façade orientale, malgré son inachèvement, à une réalisation d'apparence homogène, que son ambition rapproche des courants architecturaux contemporains, c'est à dire dans l'entourage du roi et de ses architectes.

La tradition historiographique en a attribué le projet à Jules Hardouin-Mansart. Mme de Sévigné le mentionne en effet à plusieurs reprises pendant la durée du chantier (Duchene, III, 1012, 20 octobre 1688 ; 1105, 2 mai 1689; 1207, 26 avril 1690). Mais elle ironise, elle stigmatise cette « rage (...) de bâtir et de débâtir » (Duchene, III, 1070, 14 février 1689) et 
la référence permanente à Mansart dont $\mathrm{M}$. d'Arles semble user pour justifier ses projets propres.

70 Le dessin de la façade n'est pas étranger aux productions de l'agence du premier architecte du roi dont l'influence fut considérable. D'une sobriété austère il en possède le classicisme, plus «parisien» que provençal, qu'il cite cependant avec sécheresse. La structure à avant-corps central est par ailleurs déjà celle du prieuré de Malte à Aix (1671) et du château de Barbentane (Bouches-du-Rhône, 1674), dans la lignée des œuvres des Le Vau, tous deux connus du comte de Grignan et de ses frères ${ }^{18}$.

Les croisillons et doubles croisillons sans moulure des fenêtres sont un archaïsme. On peut le penser ici dicté par souci d'harmonisation avec le motif marquant toutes les constructions, de la fin du XV $\mathrm{XV}^{\mathrm{e}}$ siècle aux années 1556-1558, expliquant l'obstination des bâtisseurs des années 1684-1689 à mettre en œuvre des baies d'un modèle parfaitement désuet. Ces doubles croisées semblaient déjà dépassées en 1648 à l'Hôtel de Ville de Tarascon et vers 1659 au château des Boyer à Eguilles, près d'Aix-en-Provence, où elles témoignent selon le terme de Jean-Jacques Gloton de la volonté méridionale d'afficher face à l'absolutisme royal un « néo-féodalisme $~{ }^{19}$.

72 La façade de l'hôtel de ville d'Arles, confié en 1673 à Hardouin-Mansart par l'entremise du coadjuteur, apporte-t-elle un point de comparaison convaincant? On y retrouve la structure à trois niveaux avec avant-corps central en avancée couronné d'un fronton triangulaire. Mais le traitement arlésien est infiniment plus savant, plus riche, plus " versaillais ", jouant avec la saillie des corniches, les balustres et les ornements sculptés, l'ordonnance de pilastres jumelés, ou de colonnes autour de la baie centrale. De manière générale la construction de Grignan fait montre d'économie, de sécheresse, sans parler de l'archaïsme des baies à croisées.

73 Parmi les œuvres de la période 1679-1687, d'autres rapprochements formels éclairent en particulier le corps central de l'édifice, seule partie présentant à vrai dire quelques caractères modernes. C'est à Versailles l'emploi analogue du bossage continu en table pour certains pavillons des écuries (1679), ou pour le corps central du grand commun (1679-1680). Les pavillons de Marly (1679-1686) associaient sur deux niveaux chaînes d'angle à bossage en table continue, double corps de moulure et fronton triangulaire, dans une composition qu'évoquent les niveaux supérieurs du corps central de Grignan. Son portail rappelle les travées inférieures de la place des Victoires (1686), avec bossage en table, arcade en plein cintre et balcon à consoles. La particularité de Grignan face à ces modèles résiderait dans la saillie du massif d'entrée destiné à recevoir le balcon, et l'accent mis sur la baie centrale à pilastres et fronton.

74 On retiendra encore le parallèle avec des dessins du fonds Robert de Cotte de la Bibliothèque nationale : ceux de la maison des pères de la Mission de Versailles (1685) ou de la façade nord du corps de logis du château de Guermantes $(1698)^{20}$ qui présentent une structure analogue à la façade de Grignan.

75 Si une parenté formelle avec l'œuvre récente de l'agence d'Hardouin-Mansart ne fait guère de doute, on ne peut évidemment pas conclure à sa paternité. En revanche le "prixfait (...) des fenestres et fassade » baillé avant le15 mai 1689 (doc. D et 0) confie clairement au maçon Jacque Jacquet dit Beaufleury la mission de modifier la façade du bâtiment de Carcassonne selon le modèle de celui d'Arles. On peut logiquement penser qu'il est aussi le maitre d'œuvre de ce dernier, ce qui expliquerait qu'il ait à payer la 
reprise de la couverture du bâtiment de Carcassonne que le nouveau chantier sous sa responsabilité aurait dégradée (doc. $\mathrm{D}$ et $\mathrm{O}$ ).

Nous l'avons dit, la présence des structures anciennes constituait une contrainte forte, d'autant plus pour les constructeurs désargentés qui ne pouvaient se permettre de transformer profondément l'édifice ou de le rebâtir a novo. Néanmoins l'absence de véritable architecte est manifeste dans le déroulement de la construction, tant du fait des archaïsmes que des incohérences relevées. Cette défaillance de la maîtrise d'ouvrage ne peut être que le fait de bâtisseurs improvisés. Et l'ironie mordante de Mme de Sévigné à l'encontre de Louis-Joseph et de Jean-Baptiste de Grignan nous en suggère les noms. Ce chantier est de ce fait un bon exemple du rôle des architectes amateurs en cette fin $\mathrm{du}$ $\mathrm{XVII}^{\mathrm{e}}$ siècle, dont le cardinal Grimaldi fournit à Aix avec son éphémère château de Puyricard l'exemple le plus spectaculaire ${ }^{21}$. Il illustre également l'effort des familles d'ancienne noblesse provençale impécunieuses pour tenir leur rang parmi celles qu'une fortune récente, acquise dans les affaires ou les hautes fonctions civiles, judiciaires ou ecclésiastiques, autorise à bâtir avec faste ${ }^{22}$.

\section{NOTES}

1. DRAS/SRA Rhône-Alpes, Lyon : Trezin, Christian, Château de Grignan. Rapports de fouille 1981-1989. La publication des recherches archéologiques sur le site est en préparation

2. Trezin, Christian. Le château de Grignan au XVI ${ }^{\mathrm{e}}$ siècle. In Congrès Archéologique (1992). Moyenne vallée du Rhône. Paris : Société française d'archéologie, 1996, p. 165-191.

3. NadaL, Abbé, Essai historique sur les Adhémar. Valence : imprimerie Marc Aurel, éditeur, 1858, pp. 197-198 et 254-256. Deves, Louis, Grignan pendant la Révolution de 1789. Montélimar : imprimerie du Progrès Astier et Niel, 1890, pp. 77-78.

4. Duchêne, Roger, Argent et famille au XVII ${ }^{\mathrm{e}}$ siècle : Mme de Sévigné et les Grignan . Provence Historique, 1965, nº 62, p. 205-228; 1966, nº 63, p. 3-41; 1966, nº6, p. 587-620.

5. Bertrand, Régis, François et Jean-Baptiste d'Adhémar de Monteil de Grignan archevêques d'Arles (1643-1689-1697). In : Duchêne, Roger (dir.), Madame de Sévigné (1626-1696). Provence, spectacles, " lanternes " [Colloque international du Tricentenaire de la mort de Madame de Sévigné, château de Grignan, 29 mai-1er juin 1996]. Grignan : A.A.C.C.D.D., 1998.

6. Boyer, Jean, Jules Hardouin-Mansart et l'hôtel de ville d'Arles. Gazette des Beaux Arts, 1969, juillet-août, p. 1-32. Voir notamment p. 29-30 le rôle du coadjuteur.

7. Duchêne, Roger, Correspondance de Mme de Sévigné. Paris: Gallimard [collection La Pléïade], t. I, 1972 ; t. II, 1974 ; t. III, 1978.

8. Dans la suite du texte nous renvoyons aux pièces de la liasse par la cotation artificielle que nous avons établie de $\mathrm{A}$ à $\mathrm{U}$, ex. : (doc. A et $\mathrm{L}$ ).

9. En 1688 il vit retiré à Grignan. AD Drôme : 2E 10423. Notaire Poumyer, fํ 68, 15 novembre 1688. 10. Soit de 248 à $270 \mathrm{~m}^{2}$ de maçonnerie (1 cane $=1,98 \mathrm{~m}$. environ).

11. CastellanE, Boni de, Mémoires de Boni de Castellane. Paris : Perrin, 1986, p. 311.

12. Inventaire du 24 février 1728.Trezin, Christian, Un palais d'Apollidon. Le château de Grignan de 1516 à 1776. Valence : Conseil général de la Drôme, 1996, p. 260. 
13. Inventaire de 1668 cité dans l'inventaire du 24 février 1728.Trezin, Christian, Un palais d'Apollidon..., p. 174-175.

14. AN : $\mathrm{T}^{*} 374$. Inventaire général des biens de Louis Adhémar, 28 mars $1597, \mathrm{f}^{\circ} 24 \mathrm{v}^{\circ}$ et $26 \mathrm{r}^{\circ}$ et $v^{\circ}$.

15. Inventaire du 24 février 1728. Trezin, Christian, Un palais d'Apollidon..., p. 262.

16. Expilly, Abbé d', Dictionnaire géographique des Gaules, 1762.

17. Information aimablement fournie par Françoise Hernandez que je remercie vivement pour sa collaboration.

18. Le marquis de Barbentane fut consul d'Aix et attaché au service du prince de Condé.

19. Gloton, Jean-Jacques, Renaissance et baroque à Aix-en-Provence. Rome : Ecole française de Rome, 1979, t.II, p. 294. Baussan Magali, Le château des Boyer, seigneurs d'Eguilles, aux XVII ${ }^{\mathbf{e}}$ et XVIII ${ }^{\mathrm{e}}$ siècles. Provence Historique, XXXIV, 140, 1985, p. 135-145.

20. Fossier, François, Les dessins du fonds Robert de Cotte de la Bibliothèque Nationale de France. Architecture et décor. Paris-Rome: Bibliothèque nationale de France/ Ecole française de Rome, BEFAR fasc. 293, 1997, p. 624, doc. 340, 1, et p. 451, doc. 211, 3.

21. Boyer, Jean, Les architectes amateurs à Aix. Provence Historique, $n^{\circ} 116,1979$. Gloton JeanJacques, Une villa italienne en Provence au XVII ${ }^{\mathrm{e}}$ siècle : le château Grimaldi de Puyricard. Provence Historique, $n^{\circ} 119,1980$, p. 5-34.

22. Gloton, Jean-Jacques, Le château méditerranéen à l'époque classique. In Babelon, JeanPierre, Le château en France. Paris : Berger-Levrault/CNMHS, 1986, p. 306.

\section{RÉSUMÉS}

La construction de l'aile "des prélats » au château de Grignan de 1684 à 1689 correspond à la requalification d'un secteur composite constitué du XII ${ }^{\mathrm{e}}$ au XVII ${ }^{\mathrm{e}}$ siècle. Commanditée sans intervention d'architecte par l'évêque de Carcassonne et l'archevêque d'Arles, frères du comte de Grignan, elle est bâtie en deux chantiers successifs selon des partis distincts dont l'harmonisation laborieuse est restée inachevée. Si le bâtiment de Carcassonne reflète initialement le savoir traditionnel de l'entrepreneur Florent Loiseleur, celui d'Arles est imprégné des œuvres récentes de l'agence de Jules Hardouin-Mansart dont le maçon Jacque Jaquet dit Beaufleury a probablement été tenu de s'inspirer. Ce chantier est un exemple du mode d'action des architectes amateurs dans le milieu conservateur de la noblesse provençale.

The "prelates" wing of the castle of Grignan was built from 1684 to 1689. It was supposed to be a requalification and a renewal of a heterogeneous area erected between 12th to 17th century. Sponsored by the bishop of Carcassonne and the archbishop of Arles, brothers of the count of Grignan, no architect was involved in its design. It was built in two successive phases reflecting distinct conceptions and accordingly its architctural harmonization was made difficult and remained unfulfilled. If the Carcassonne building reflects the traditional savoir faire of the building contractor Florent Loiseleur, Arles's was deeply close to the recent works of Jules Hardouin-Mansart's agency whom the master builder Jacque Jaquet, said Beaufleury, had most probably to be inspired of. This construction is an example of the way amateur architects used to work in the conservative circle of provençal nobility. 
INDEX

Mots-clés : inventaire général, en ligne, journal, revue électronique, revue numérique, périodique, patrimoine, histoire de l'art, France, château, architecture domestique, Rhône-Alpes Keywords : on line, electronic journal, ejournal, heritage, history of art, architecture, country house

\section{AUTEUR}

\section{CHRISTIAN TRÉZIN}

Conservateur régional de l'Inventaire général du Centre, DRAC Centre, 6 rue de la Manufacture 45043 Orléans. christian.trezin@culture. gouv.fr 